\title{
Evolution of H9N2 influenza viruses isolated in Shandong Province
}

\author{
ZHANG Wei ${ }^{1,2 \dagger}$, XU HuaiYing ${ }^{2 \dagger}$, MENG Fang ${ }^{4}$, MA XiuLi ${ }^{2}$, LIU Xia $^{2}$, HUANG DiHai ${ }^{2}$, \\ QIN ZhuoMing ${ }^{2,3^{*}}$, LIU JinHua $^{1 *} \&$ ZHAO Peng ${ }^{4 *}$ \\ ${ }^{1}$ College of Veterinary Medicine, China Agricultural University, Beijing 100193, China; \\ ${ }^{2}$ Shandong Academy of Agricultural Science, Jinan 250100, China; \\ ${ }^{3}$ Shandong Jianmu Biological Pharmaceutical Co., Ltd, Jinan 250100, China; \\ ${ }^{4}$ College of Animal Science and Veterinary Medicine, Shandong Agricultural University, Tai'an 271018, China
}

Received March 9, 2014; accepted August 18, 2014; published online January 6, 2015

Citation: Zhang W, Xu HY, Meng F, Ma XL, Liu X, Huang DH, Qin ZM, Liu JH, Zhao P. Evolution of H9N2 influenza viruses isolated in Shandong Province. Sci China Life Sci, 2015, 58: 212-214, doi: 10.1007/s11427-014-4777-0

\section{Dear Editor,}

The low-pathogenic avian influenza subtype of the H9N2 virus circulates in domestic poultry and wild birds throughout the world, causing severe morbidity and mortality in commercial chickens during coinfection with other pathogens, resulting in enormous losses. This kind of virus has been prevalent since the H9N2 virus was first identified in China in 1994.

The complete genome of the H9N2 virus is composed of eight negative-sense single-stranded RNA fragments. The hemagglutinin (HA) protein, the major component of the spikes on the surface of the viral envelope, is closely related to the antigenicity of the virus, including its hemagglutination activity and neutralizing antibodies. HA is also recognized as one of the critical factors in the efficacy of influenza $A$ vaccines $[1,2]$. Our aim was to comprehensively clarify the antigenic, genetic, and evolutionary characteristics of H9N2 isolates from Shandong Province. The 35 most prevalent H9N2 strains in the area from 1999 to 2013 were investigated.

The phylogenetic tree of HA gene segments is shown in Figure 1 and reveals that three H9N2 isolates, including JN05, belong to the BJ94-like strains, which constituted the

$\dagger$ Contributed equally to this work

*Corresponding author (email: qinzm1997@163.com; 1jh@cau.edu.cn; zhaopeng@sdau.edu.cn) predominate lineage from 1994 to 2005; and four viruses, including JZ00, isolated from 1999 to 2008, belong to the Y280-like sublineage. Twenty-eight H9N2 viruses isolated in 2010-2013 belong to the S2-like subgenotype, suggesting that this genotype has been the dominant H9N2 strain in Shandong Province in recent years. Interestingly, a comparison of homologies showed that the strains isolated in 2010-2013 shared higher homology than did the strains from other years. The H9N2 viruses isolated in 2010-2013 share nucleotide homologies (amino acid) of 94.5\%-100\% $(96.1 \%-100 \%)$, but only homologies of $90.0 \%-92.5 \%$ $(91.8 \%-95.0 \%)$ with the vaccine strain SD-6 isolated in 1996.

An analysis of the key amino acid sites showed that the HA proteins of the H9N2 strains isolated after 2010 share the cleavage site motif PSRSSR $\downarrow$ GL and contain an A-to-S substitution at residue 334. Moreover, some H9N2 viruses, with a leucine residue at amino acid 226 (numbered by H3) in the hemagglutinin (HA) receptor-binding site (RBS), display human-virus-like receptor specificity [3]. These results indicate that the H9N2 viruses pose a public health risk and might have pandemic potential in the future.

Immunization with effective inactivated vaccines is considered a key strategy and plays a crucial role in preventing and controlling H9N2 avian influenza. However, the rapid antigenic evolution of these viruses and the mismatches between different H9N2 isolates allow them to readily es- 


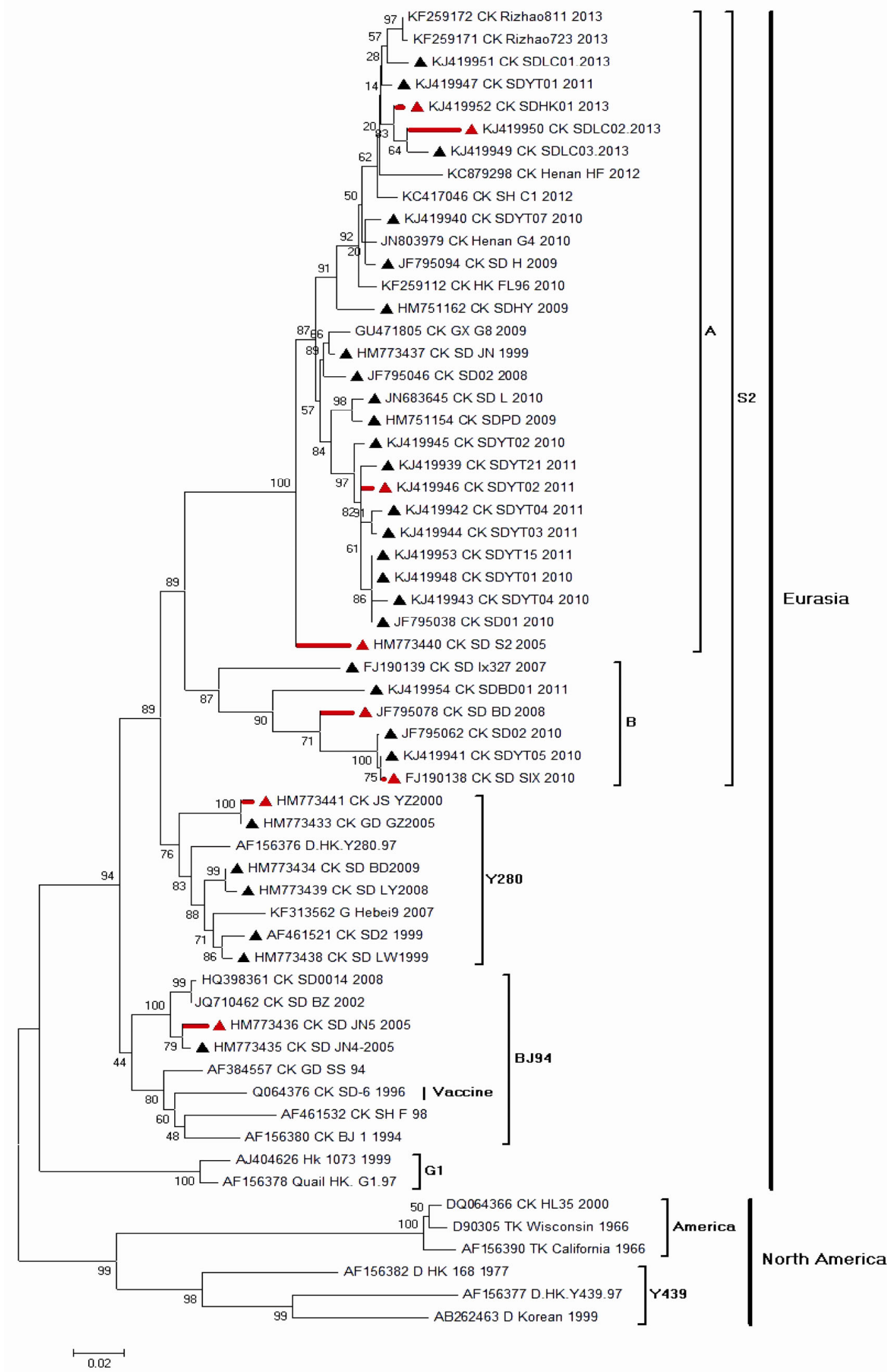

Figure 1 Phylogenetic tree based on the nucleotide sequences of the HA genes of H9N2 isolates and reference strains selected from GenBank. $\boldsymbol{\Delta}$ indicates $\mathrm{H} 9 \mathrm{~N} 2$ isolates determined in this study; red indicates isolates used in an $\mathrm{HI}$ assay. 
cape population immunity [4,5]. In this study, to clarify the relationships among the $\mathrm{H} 9 \mathrm{~N} 2$ isolates belonging to distinct sublineages, eight H9N2 were selected according to their genotypes based on the $H A$ gene and the results of a cross-hemagglutinin-inhibition (HI) assay. Both the $\mathrm{R}$ values and the amino acid homologies are shown in Table S1 in Supporting Information. The results indicate that there is a close relationship among all eight strains. A significant level of HI was observed between the strains, and most of the HI correlation indices $(R)$ exceeded 0.31 . Interestingly, BD08 (isolated in 2008), the prevailing strain in China, shared $R$ values with recent $\mathrm{H} 9 \mathrm{~N} 2$ isolates ranging from 0.57 to 1.0 , higher than those of fresh isolates in different years. However, LC02/13 and HK01/12 shared 97.3\% amino acid homology, whereas their $R$ value was only 0.31 , the lowest among these isolates. These data suggest that there is some antigenic discrepancy between the vaccine-like strain and the field isolates.

Collectively, these data suggest that immune pressure, resulting from widespread H9N2 vaccination in China, has increased the antigenic variation among the H9N2 strains isolated in recent years.

1 Wang YL, Yuan XL, Xu HY, Zhang YX, Qin ZM. Identification and molecular characterization of a $\mathrm{H} 9 \mathrm{~N} 2$ isolate from a broiler flock in high antibody level against H9N2. Acta Agricult Boreali Sin, 2010, 25: 23-27

2 Zhang P, Tang Y, Liu X, Peng D, Liu W, Liu H, Lu S, Liu X. Characterization of H9N2 influenza viruses isolated from vaccinated flocks in an integrated broiler chicken operation in eastern China during a 5 year period (1998-2002). J Gen Virol, 2008, 89(Pt 12): 3102-3112

3 Zhang P, Tang Y, Liu X, Liu W, Zhang X, Liu H, Peng D, Gao S, Wu Y, Zhang L, Lu S, Liu X. A Novel genotype H9N2 influenza virus possessing human $\mathrm{H} 5 \mathrm{~N} 1$ internal genomes has been circulating in poultry in eastern China since 1998. J Virol, 2009, 83: 8428-8438

4 Li C, Yu K, Tian G, Yu D, Liu L, Jing B, Ping J, Chen H. Evolution of H9N2 influenza viruses from domestic poultry in Mainland China. Virology, 2005, 340: 70-83

5 Lee YJ, Shin JY, Song MS, Lee YM, Choi JG, Lee EK, Jeong OM, Sung HW, Kim JH, Kwon YK, Kwon JH, Kim CJ, Webby RJ, Webster RG, Choi YK. Continuing evolution of H9 influenza viruses in Korean poultry. Virology, 2007, 359: 313-323

Open Access This article is distributed under the terms of the Creative Commons Attribution License which permits any use, distribution, and reproduction in any medium, provided the original author(s) and source are credited.

\section{Supporting Information}

Table S1 Correlation indices for hemagglutination inhibition and homologies in the amino acid sequences of the hemagglutinin genes of H9N2 strains isolated in Shandong Province

The supporting information is available online at life.scichina.com and link.springer.com. The supporting materials are published as submitted, without typesetting or editing. The responsibility for scientific accuracy and content remains entirely with the authors. 